\title{
When routine 7-day ambulatory ECG monitoring should be advocated in patients with ischaemic stroke
}

\author{
Authors: Alistair Mackett, Muhibbur Chowdhury and Saugata Das
}

\section{Aims}

Stroke associated with atrial fibrillation (AF) carries a more unfavourable prognosis, and current screening guidance suggests electrocardiogram (ECG) monitoring for at least 24 hours. It should be noted, however, that 24 -hour monitoring in cryptogenic strokes has relatively low rates of AF detection (1.4-6\%). Prolonged ECG monitoring can demonstrate that a significant proportion of 'cryptogenic strokes' are in fact related to paroxysmal atrial fibrillation ( $\mathrm{pAF}$ ). Our physicians until now advocated the use of 24-hour monitors in all cases of ischaemic stroke and selected cases of TIA. Our aim was to ascertain our current rates of AF detection and to identify where prolonged recording resources should be directed.

\section{Methods}

All ambulatory ECG investigations completed under the stroke physicians at a large district general hospital over a 6-month period (January-June 2014) were reviewed. Every patient's electronic notes were reviewed, including their diagnosis and ambulatory ECG result.

\section{Results}

214 tapes were completed (178 stoke/TIA, 36 non-stroke). Overall AF rates were $8.9 \%$ in stroke patients with a new diagnosis rate of $6.7 \%$. We stratified diagnosis of stroke by Bamford classification.

$\begin{array}{llll}\text { Diagnosis } & \text { Number }(\mathbf{n}=178) & \text { New diagnosis AF } & \text { Total AF } \\ \text { Total anterior circulation infarct (TACI) } & 11(6 \%) & 2(22 \%) & 4(36 \%) \\ \text { Partial anterior circulation infarct (PACI) } & 54(30 \%) & 6(11.5 \%) & 7(13 \%) \\ \text { Posterior circulation infarct (POCI) } & 20(11 \%) & 2(10 \%) & 2(10 \%) \\ \text { Lacunar infarct (LACI) } & 34(19 \%) & 1(3 \%) & 2(6 \%) \\ \text { Transient ischaemic attack (TIA) } & 60(34 \%) & 1(2 \%) & 1(2 \%)\end{array}$

\begin{abstract}
Conclusions
Our data reflected relatively low detection rates of pAF from 24-hour ECGs in LACI and TIA, especially with an unremarkable baseline ECG. From a risk stratification viewpoint, individuals with anterior circulation strokes with cortical involvement (PACI, TACI) without obvious evidence of large vessel atherosclerotic disease, posterior circulation strokes and those involving more than one vascular territory will be recommended to have 7-day monitoring as routine. Subsequent detection of pAF led to $>97 \%$ patients in CRYSTAL-AF commencing on anticoagulation. We anticipate the change to be cost neutral as the total number of requests should decrease. Evaluation of the effectiveness and yield of pAF of the 7-day monitors in the proposed selected cases will be undertaken.
\end{abstract}

